\title{
Bone and ocular safety of budesonide/ glycopyrrolate/formoterol fumarate metered dose inhaler in COPD: a 52-week randomized study
}

\author{
Edward M. Kerwin ${ }^{1 *}$, Gary T. Ferguson ${ }^{2}$, Mindy $\mathrm{Mo}^{3}$, Kiernan DeAngelis ${ }^{4}$ and Paul Dorinsky ${ }^{4}$
}

\begin{abstract}
Background: Long-term use of inhaled corticosteroids (ICSs) has been associated with increased risk of bone and ocular comorbidities. We evaluated the effects of the triple fixed-dose combination budesonide/ glycopyrrolate/formoterol fumarate metered dose inhaler (BGF MDI), formulated using co-suspension delivery technology, on bone mineral density (BMD) and ocular safety in patients with moderate-tovery severe chronic obstructive pulmonary disease (COPD).

Methods: In this extension study, a subset of patients from the 24-week, phase III, randomized, doubleblind KRONOS study (NCT02497001) continued treatment (BGF MDI 320/18/9.6 $\mu$ g, budesonide/formoterol fumarate [BFF] MDI 320/9.6 $\mu \mathrm{g}$ or glycopyrrolate/formoterol fumarate [GFF] MDI 18/9.6 $\mathrm{mg}$, as a non-steroidal comparator) for an additional 28 weeks. Primary endpoints were percentage change from baseline in lumbar spine BMD and change from baseline in lens opacities classification system III posterior subcapsular cataract (P) score, both at Week 52. Adverse events were also assessed.

Results: In total, 456 patients were included in the safety population (53.1\% male, mean age 62.8 years). Changes from baseline in lumbar spine BMD (least squares mean [LSM] range -0.12 to $0.38 \%$ ) and $P$ score (LSM range 0.02-0.15) were small for all treatments. Both BGF MDI and BFF MDI were non-inferior to GFF $\mathrm{MDI}$ using margins of $-2 \%(B M D)$ and 0.5 units ( $P$ score). The incidence of treatment-emergent adverse events (TEAEs) was generally similar among groups. Rates of confirmed pneumonia were low overall (2.4\%) and highest in the GFF MDI group (3.4\%), followed by BGF MDI (2.1\%) and BFF MDI (1.1\%). There were no cumulative adverse effects of treatment over time as the incidence and types of TEAEs, were generally similar in the first 24 weeks of the study and after Week 24.

Conclusions: In patients with COPD, both ICS-containing therapies were non-inferior to GFF MDI for the primary BMD and ophthalmological endpoints. Changes from baseline in all three treatment groups over 52 weeks were small and not clinically meaningful. All treatments were well tolerated with no new or unexpected safety findings.
\end{abstract}

Trial registration: ClinicalTrials.gov NCT02536508. Registered 27 August 2015.

Keywords: BGF MDI, Bone mineral density, Inhaled corticosteroid, Lens opacity, LOCS III, Metered dose inhaler

\footnotetext{
* Correspondence: ekerwin@criresearch.com

${ }^{1}$ Clinical Research Institute of Southern Oregon, 3860 Crater Lake Avenue,

Medford, OR 97504, USA

Full list of author information is available at the end of the article
}

(c) The Author(s). 2019 Open Access This article is distributed under the terms of the Creative Commons Attribution 4.0 International License (http://creativecommons.org/licenses/by/4.0/), which permits unrestricted use, distribution, and reproduction in any medium, provided you give appropriate credit to the original author(s) and the source, provide a link to the Creative Commons license, and indicate if changes were made. The Creative Commons Public Domain Dedication waiver (http://creativecommons.org/publicdomain/zero/1.0/) applies to the data made available in this article, unless otherwise stated. 


\section{Introduction}

Treatment with long-acting bronchodilators is central to the management of chronic obstructive pulmonary disease (COPD) [1]. For patients receiving dual longacting bronchodilator therapy who experience continued COPD exacerbations, the addition of an inhaled corticosteroid (ICS) is a recommended treatment option [1], and triple therapies have been shown to improve lung function and quality of life, and reduce exacerbations versus corresponding dual long-acting bronchodilator therapies [2, 3]. However, concerns remain regarding possible consequences of long-term ICS use, including decreased bone density and the development of cataracts. These are especially relevant for patients with COPD as the majority are older adults [4], and risk of bone loss and ocular comorbidities increases with age $[5,6]$.

Furthermore, the incidence of osteoporosis and glaucoma is increased in patients with COPD even compared with age-matched controls [7], which may result from a combination of factors including corticosteroid use (oral and/or inhaled), smoking, and physiological complications of COPD including hypoxia and systemic inflammation [8-10]. Studies of ICS use that have investigated the risk of bone- and ocular-related adverse effects have varied in their findings [11-19], potentially due to differences in study duration, patient population, ICS compound used, and dose. Therefore, it is important to evaluate the impact of novel ICS-containing therapies on the incidence of these potential adverse effects.

Budesonide/glycopyrrolate/formoterol fumarate metered dose inhaler (BGF MDI), an ICS/long-acting anti-muscarinic antagonist (LAMA)/long-acting $\beta_{2}$-agonist (LABA) triple fixed-dose combination (FDC) formulated using cosuspension delivery technology, had benefits on lung function, symptoms and exacerbations versus dual therapies in a pivotal 24-week, phase III study in patients with moderate-to-very severe COPD (KRONOS) [2]. To further assess the tolerability of BGF MDI over a longer treatment period, a subset of patients who participated in KRONOS continued into an extension study to examine the effects of triple therapy with BGF MDI and the corresponding dual therapies budesonide/formoterol fumarate (BFF) MDI and glycopyrrolate/formoterol fumarate (GFF) MDI on bone mineral density (BMD) and ocular safety over 52 weeks.

\section{Methods}

\section{Study design and treatment}

This was an extension study (registered with ClinicalTrials. gov; NCT02536508) conducted in a subset of patients from US sites who were initially enrolled in the 24-week KRONOS study (NCT02497001) and continued their randomized treatment for 28 additional weeks (Additional file 1: Figure S1). The KRONOS study was a randomized, double-blind, parallel-group, multicenter, phase III study in which patients with moderate-to-very severe COPD were randomized 2:2:1:1 to BGF MDI 320/18/9.6 $\mu \mathrm{g}$, GFF MDI $18 / 9.6 \mu \mathrm{g}$, BFF MDI 320/9.6 $\mu \mathrm{g}$, or open-label budesonide/ formoterol fumarate dihydrate dry powder inhaler (DPI) 400/12 $\mu \mathrm{g}$ (Symbicort ${ }^{\oplus}$ Turbuhaler $^{\circledR}$ ) as an active control (all via two oral inhalations, twice daily) [2]. Details of the randomization have been described [2]. Glycopyrrolate $18 \mu \mathrm{g}$ and formoterol fumarate $9.6 \mu \mathrm{g}$ are equivalent to $14.4 \mu \mathrm{g}$ of glycopyrronium and $10 \mu \mathrm{g}$ of formoterol fumarate dihydrate. Patients randomized to budesonide/formoterol DPI in KRONOS were not enrolled in the extension study.

The entire 52-week study took place from 24 September 2015 to 6 September 2017. The informed consent form and study protocol were approved by an institutional review board (Schulman Associates; approval number 201503097), and patients provided written informed consent for both KRONOS and the extension study prior to screening. The study was conducted in accordance with the Declaration of Helsinki and the International Conference on Harmonisation/Good Clinical Practice, as well as applicable regulatory requirements.

\section{Study population}

The inclusion/exclusion criteria for KRONOS [2] also applied to the extension study. Briefly, patients were 4080 years of age with an established clinical history of COPD, a smoking history of $\geq 10$ pack-years and a postbronchodilator forced expiratory volume in $1 \mathrm{~s}\left(\mathrm{FEV}_{1}\right) /$ forced vital capacity ratio $<0.70$ and post-bronchodilator $\mathrm{FEV}_{1}<80 \%$ and $\geq 25 \%$ predicted normal value. Exclusion criteria specific to the 52-week study comprised severe osteoporosis, a T-score $<-2.5$ at baseline or inability to achieve an acceptable BMD scan (BMD exclusion criteria); and inability to dilate pupil $\geq 6 \mathrm{~mm}$, intraocular pressure $(\mathrm{IOP}) \geq 21 \mathrm{mmHg}$ (lowest of 3 readings), or an implanted artificial intraocular lens (ophthalmological exclusion criteria). Important protocol amendments are shown in Additional file 1: Table S1.

\section{Bone and ocular safety endpoints}

BMD endpoints included the percentage change from baseline in BMD of lumbar spine segments 2-4 (L2-L4) at Week 52 (primary BMD endpoint) and percentage change from baseline in BMD of the total hip at Week 52 (other BMD endpoint). BMD was evaluated at baseline (Day 1 of the KRONOS study, prior to randomization) and Week 52 using dual-energy x-ray absorptiometry, with two scans taken at each site.

The primary ophthalmological endpoint was the change from baseline (assessed during screening in KRONOS) in the lens opacities classification system III (LOCS III) posterior subcapsular cataract (P) 
score at Week 52. Other ophthalmological endpoints (all at Week 52) included: changes from baseline in LOCS III cortical cataract (C), nuclear color (NC), and nuclear opalescence (NO) scores; changes from baseline in IOP, logarithm of the minimum angle of resolution (LogMAR) visual acuity scores, and horizontal cup-to-disc ratio; the proportion of patients with LOCS III grade increases of $\geq 0.5, \geq 1.0$, or $\geq 1.5$ units in each of the 4 scales; and the proportion of patients with IOP $\geq 22 \mathrm{mmHg}$ or change from baseline in IOP of $\geq 7 \mathrm{mmHg}$. Further details regarding the bone and ocular safety assessments are provided in Additional file 1: supplementary methods.

\section{Additional safety evaluations}

Safety was additionally assessed by adverse event (AE) monitoring, 12-lead electrocardiography, clinical laboratory testing, and vital sign measurements. Adverse events of special interest were identified based on the pharmacologically predictable effects of ICSs, LAMAs, and LABAs (further details are provided in Additional file 1: supplementary methods). Cases of pneumonia and major adverse cardiovascular events (MACE) were reviewed and adjudicated by an external, independent Clinical Endpoint Committee against predefined criteria.

\section{Efficacy endpoints}

Efficacy endpoints assessed over 52 weeks included the change from baseline in average daily rescue salbutamol use, percentage of days with no rescue salbutamol use, rate of moderate/severe COPD exacerbations, and change from baseline in Exacerbations of Chronic Pulmonary Disease Tool (EXACT) and EXACT-Respiratory Symptom (RS) total scores. Patients continued to use an electronic diary provided during KRONOS to record study medication use, the total daily number of 'puffs' of rescue medication, COPD symptoms, and responses for EXACT (a 14-item questionnaire assessing patient-reported outcomes [20]).

\section{Statistical analysis}

The safety and modified intent-to-treat populations included all patients who signed the informed consent form for the extension study and received any amount of study drug (other than budesonide/formoterol DPI), excluding those who did not meet eligibility criteria at baseline or had no data collected after the Week 24 transition visit. The extension study safety population was defined as all patients in the safety population who met the continued eligibility criteria for the extension study at the Week 24 transition visit or attended at least one visit during Weeks 28-52 (Additional file 1: Figure S1). The BMD and ophthalmological populations were defined as all evaluable patients in the safety population who had baseline and $\geq 1$ on-treatment BMD or ophthalmological assessments, respectively, analyzed according to actual treatment received.

For the primary BMD endpoint, non-inferiority was declared if the lower 95\% confidence interval (CI) bound for the percentage treatment difference in lumbar spine BMD was $>-2 \%$. Non-inferiority for the primary ophthalmological endpoint was declared if the upper $95 \% \mathrm{CI}$ bound for the treatment difference in LOCS III P scores was $<0.5$ units. Because the primary objectives were related to safety, all hypotheses were tested at nominal alphas, and there were no controls for multiplicity. Further details are provided in Additional file 1: supplementary methods.

\section{Results}

\section{Study population}

In total, 627 patients who were randomized in KRONOS consented to participate in the extension study (Fig. 1). Of these, 169 patients (27.0\%) did not meet the extension study entry criteria at baseline and lacked post-Week 24 transition visit data (including 81 patients [12.9\%] who had BMD T-scores $<-2.5$ at baseline), and were excluded from the study analysis populations. Seventy-two patients who had consented to participate in the extension study when they entered KRONOS discontinued treatment prior to Week 24. A quarter of these patients $(n=18)$ discontinued due to AEs, none of which were bone- or ocular-related (further details are provided in Additional file 1: supplementary results). The safety population included $456 \mathrm{pa}-$ tients $(53.1 \%$ male, mean age of 62.8 years; Table 1$)$. Demographic characteristics were generally similar across treatment groups. Mean study drug exposure was comparable across groups, ranging from 298.2 days (BFF MDI) to 310.4 days (GFF MDI). The percentage of patients with exposure $\geq 24$ weeks (range 81.8 to $86.2 \%$ ) and $\geq 48$ weeks (range 72.2 to $75.0 \%$ ) was similar across the treatment groups.

\section{BMD endpoints}

Baseline BMD characteristics were comparable across treatment groups (Table 2), and the majority of patients had normal BMD (T-scores >-1) at the lumbar spine (72.0\%) and hip (59.9\%). The mean T-scores in the BMD population at baseline were 0.1 for lumbar spine BMD and -0.6 for hip BMD, which were comparable to those in patients who withdrew from the study prior to Week 24 (0.0 and -0.7 , respectively).

For the primary BMD endpoint, percentage changes from baseline at Week 52 were small (least squares mean [LSM] range -0.12 to $0.38 \%$ ) and similar across treatment groups (Table 2; Additional file 1: Figure S2) and both ICS-containing treatments were non-inferior 


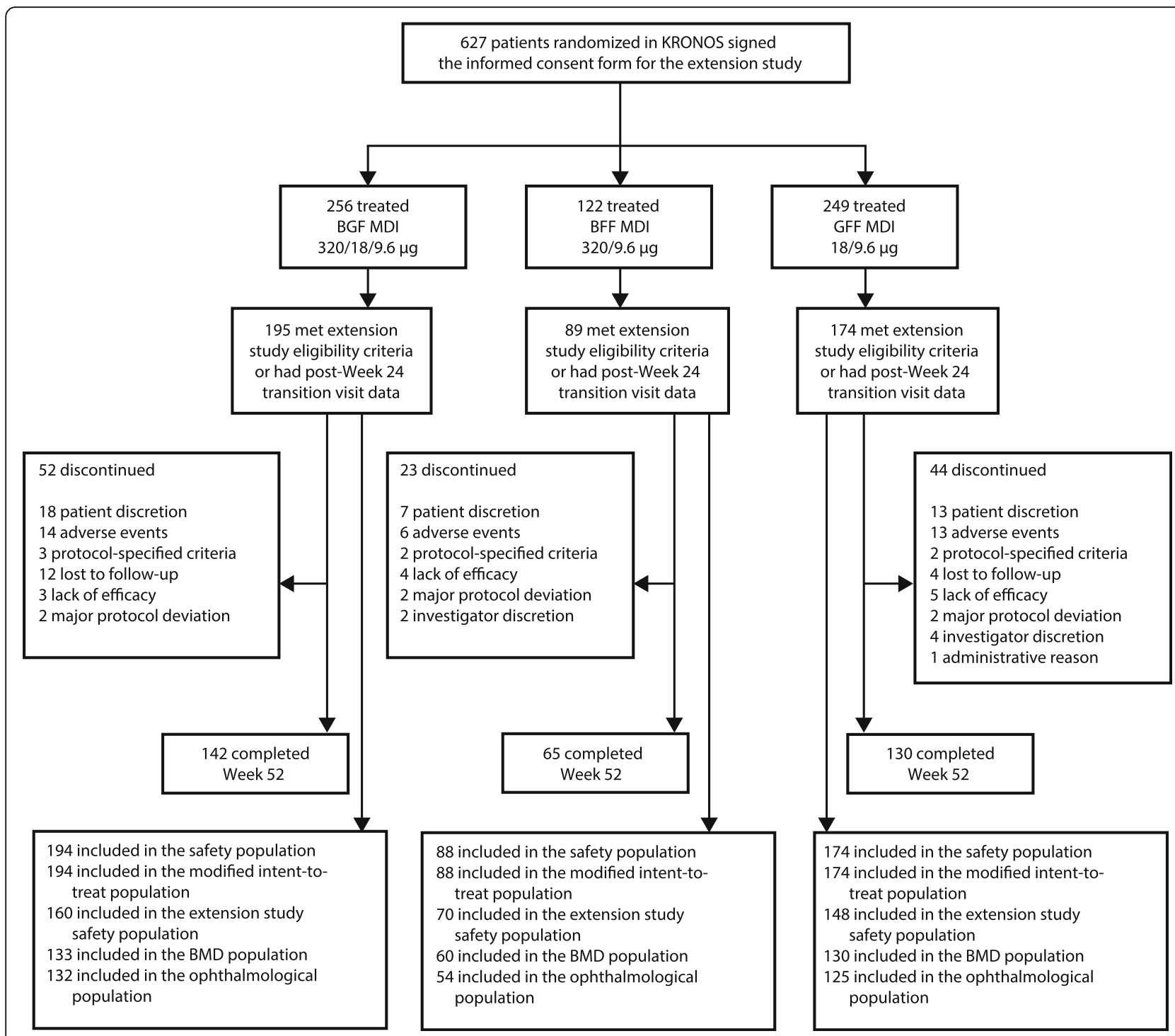

Fig. 1 Patient disposition. Two patients (one in the BGF MDI group and one in the BFF MDI group) participated in multiple sponsor-led studies and were excluded from all analysis populations. BFF Budesonide/formoterol fumarate, BGF Budesonide/glycopyrrolate/formoterol fumarate, BMD Bone mineral density, GFF Glycopyrrolate/formoterol fumarate, MDI Metered dose inhaler

to GFF MDI. The percentage changes from baseline in total hip BMD (other BMD endpoint) were also small (LSM range -1.12 to $-0.32 \%$ ) and comparable for all three treatments (Table 2, Additional file 1: Figure S2).

T-scores were calculated at baseline and Week 52 to assess shifts in BMD status during the treatment period (Table 3). Lumbar spine T-scores worsened from normal $(>-1)$ to osteopenic $(>-2.5$ and $\leq-1)$ for $3.1,5.3$, and $3.3 \%$ of patients treated with BGF MDI, BFF MDI, and GFF MDI, respectively (Table 3). No patients shifted to a lumbar spine BMD T-score of $\leq-2.5$ (indicative of osteoporosis). Improvements in lumbar spine $\mathrm{T}$-scores were observed for 1.6, 3.5, and $7.3 \%$ of patients treated with BGF MDI, BFF MDI, and GFF MDI, respectively. For total hip
BMD, 2.3\% (BGF MDI), 1.8\% (BFF MDI), and $0.8 \%$ (GFF MDI) of patients shifted from an osteopenic to osteoporotic T-score; and 3.9, 8.8, and $3.4 \%$ of patients, respectively, shifted from normal to osteopenic (Table 3). In the BGF MDI, BFF MDI, and GFF MDI groups, 3.1, 1.8, and $0.8 \%$ of patients, respectively, improved from osteopenic at baseline to a normal T-score at Week 52.

\section{Ophthalmological endpoints}

At baseline, most patients ( $>94 \%$ overall) had normal horizontal cup-to-disc ratio (mean 0.3, range 0.0-1.0) and IOP (mean 14.9, range 8.0-25.0) and were not taking ophthalmological medications as eye drops. Baseline ophthalmological characteristics were similar across treatment 
Table 1 Baseline demographics and clinical characteristics (safety population)

\begin{tabular}{|c|c|c|c|}
\hline & $\begin{array}{l}\text { BGF MDI } \\
320 / 18 / 9.6 \mu \mathrm{g} \\
(N=194)\end{array}$ & $\begin{array}{l}\text { BFF MDI } \\
320 / 9.6 \mu \mathrm{g} \\
(N=88)\end{array}$ & $\begin{array}{l}\text { GFF MDI } \\
18 / 9.6 \mu \mathrm{g} \\
(N=174)\end{array}$ \\
\hline Age, years (mean [SD]) & $62.6(7.9)$ & $64.0(7.2)$ & $62.4(7.8)$ \\
\hline Male, n (\%) & $102(52.6)$ & $53(60.2)$ & $87(50.0)$ \\
\hline \multicolumn{4}{|l|}{ Race, n (\%) } \\
\hline White & $179(92.3)$ & 79 (89.8) & $156(89.7)$ \\
\hline Black & $13(6.7)$ & $9(10.2)$ & $17(9.8)$ \\
\hline Other & $2(1.0)$ & 0 & $1(0.6)$ \\
\hline \multicolumn{4}{|l|}{ Ethnicity, n (\%) } \\
\hline Hispanic or Latino & $9(4.6)$ & $6(6.8)$ & $5(2.9)$ \\
\hline Not Hispanic or Latino & $185(95.4)$ & $82(93.2)$ & $169(97.1)$ \\
\hline Body mass index, $\mathrm{kg} \cdot \mathrm{m}^{-2}$ (mean [SD]) & $29.0(7.4)$ & $29.0(5.8)$ & $29.0(6.5)$ \\
\hline Current smoker, $n(\%)$ & $101(52.1)$ & $42(47.7)$ & $95(54.6)$ \\
\hline $\begin{array}{l}\text { Number of pack-years smoked }{ }^{\#} \\
\text { (median [range]) }\end{array}$ & $45.0(11.2-256.0)$ & $47.3(14.3-134.0)$ & $50.0(10.0-171.0)$ \\
\hline \multicolumn{4}{|l|}{ COPD severity, $n(\%)$} \\
\hline Moderate & $95(49.0)$ & $45(51.1)$ & $91(52.3)$ \\
\hline Severe & $86(44.3)$ & $37(42.0)$ & $65(37.4)$ \\
\hline Very severe & $13(6.7)$ & $6(6.8)$ & $18(10.3)$ \\
\hline COPD duration, years (mean [SD]) & $8.6(6.7)$ & $9.6(6.3)$ & $7.7(5.3)$ \\
\hline \multicolumn{4}{|l|}{$\begin{array}{l}\text { Moderate/severe COPD } \\
\text { exacerbations in } \\
\text { the past } 12 \text { months, } n(\%)\end{array}$} \\
\hline 0 & $152(78.4)$ & $67(76.1)$ & $129(74.1)$ \\
\hline 1 & $33(17.0)$ & $18(20.5)$ & $34(19.5)$ \\
\hline$\geq 2$ & $9(4.6)$ & $3(3.4)$ & $11(6.3)$ \\
\hline $\begin{array}{l}\text { Eosinophil count, cells } \cdot \mathrm{mm}^{-3} \\
\text { (median, [range]) }\end{array}$ & $180(10-655)$ & $190(15-505)$ & $185(40-2490)$ \\
\hline$<150$ cells $\cdot \mathrm{mm}^{-3}, n(\%)$ & $68(35.1)$ & $32(36.4)$ & $54(31.0)$ \\
\hline$\geq 150$ cells $\cdot \mathrm{mm}^{-3}, n(\%)$ & $126(64.9)$ & $56(63.6)$ & $120(69.0)$ \\
\hline Use of ICS at screening, $n$ (\%) & $152(78.4)$ & $73(83.0)$ & $127(73.0)$ \\
\hline CAT total score (mean [SD]) & $\begin{array}{l}21.2(6.4) \\
n=192\end{array}$ & $\begin{array}{l}22.3(6.7) \\
n=86\end{array}$ & $\begin{array}{l}20.4(6.3) \\
n=172\end{array}$ \\
\hline EXACT total score (mean [SD]) & $\begin{array}{l}35.2(10.8) \\
n=194\end{array}$ & $\begin{array}{l}35.7(10.4) \\
n=86\end{array}$ & $\begin{array}{l}34.6(10.6) \\
n=174\end{array}$ \\
\hline 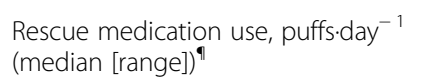 & $\begin{array}{l}1.9(0.0-12.0) \\
n=194\end{array}$ & $\begin{array}{l}2.4(0.0-17.7) \\
n=86\end{array}$ & $\begin{array}{l}2.0(0.0-18.2) \\
n=174\end{array}$ \\
\hline
\end{tabular}

BFF Budesonide/formoterol fumarate, BGF Budesonide/glycopyrrolate/formoterol fumarate, CAT COPD Assessment Test, COPD Chronic obstructive pulmonary disease, EXACT Exacerbations of Chronic Pulmonary Disease Tool, GFF Glycopyrrolate/formoterol fumarate, ICS Inhaled corticosteroid, MDI Metered dose inhaler, $\mathrm{m} / T$ Modified intent-to-treat, $S D$ Standard deviation. ": number of pack-years smoked $=($ number of cigarettes each day/20) $\times$ number of years smoked, ": mITT population

groups (Table 4), although the GFF MDI group had slightly lower LOCS III scores at baseline compared to the BGF MDI group. Changes from baseline in LOCS III (P) score at Week 52 (primary ophthalmological endpoint) were small (LSM range 0.02-0.15) and similar among treatment groups (Table 4, Additional file 1: Figure S3); BGF MDI was non-inferior to GFF MDI. Similarly, changes from baseline in LOCS III NO, NC, and C scores at Week 52 were small (LSM $\leq 0.26$ ), with the upper bound of the $95 \%$ CI consistently below the clinically meaningful value of 0.5 for BGF MDI versus GFF MDI (Table 4, Additional file 1: Figure S3). Changes from baseline in IOP at Week 52 were small and similar in the BGF MDI $(0.67 \mathrm{mmHg})$ and GFF MDI $(0.68 \mathrm{mmHg})$ treatment groups (Table 4), below the clinically meaningful value of $2 \mathrm{mmHg}$. There were no changes from baseline (in both 
Table 2 Primary and other BMD endpoints (BMD population)

\begin{tabular}{|c|c|c|c|}
\hline & $\begin{array}{l}\text { BGF MDI } \\
320 / 18 / 9.6 \mu \mathrm{g} \\
(N=133)\end{array}$ & $\begin{array}{l}\text { BFF MDI } \\
320 / 9.6 \mu g \\
(N=60)\end{array}$ & $\begin{array}{l}\text { GFF MDI } \\
18 / 9.6 \mu \mathrm{g} \\
(N=130)\end{array}$ \\
\hline \multicolumn{4}{|l|}{ Lumbar spine BMD (L2-L4) } \\
\hline$n$ & 128 & 57 & 123 \\
\hline Baseline, $\mathrm{g} \cdot \mathrm{cm}^{-2}$ (mean [SD]) & $1.18(0.21)$ & $1.19(0.20)$ & $1.16(0.18)$ \\
\hline \multicolumn{4}{|c|}{$\begin{array}{l}\text { Change from baseline at Week 52, \% } \\
\text { (primary BMD endpoint) }\end{array}$} \\
\hline LSM & -0.09 & -0.12 & 0.38 \\
\hline $95 \% \mathrm{Cl}$ & $(-0.74,0.56)$ & $(-1.09,0.86)$ & $(-0.28,1.04)$ \\
\hline \multicolumn{4}{|c|}{ LSM \% difference between treatments } \\
\hline \multicolumn{4}{|l|}{ Versus GFF MDI 18/9.6 $\mu \mathrm{g}^{\#}$} \\
\hline LSM & -0.47 & -0.50 & NA \\
\hline $95 \% \mathrm{Cl}$ & $(-1.38,0.45)$ & $(-1.69,0.68)$ & \\
\hline \multicolumn{4}{|c|}{ Versus BFF MDI 320/9.6 $\mu g^{\prime \prime}$} \\
\hline LSM & 0.03 & NA & Shown above \\
\hline $95 \% \mathrm{Cl}$ & $(-1.13,1.20)$ & & \\
\hline \multicolumn{4}{|l|}{ Total hip BMD } \\
\hline$n$ & 128 & 57 & 119 \\
\hline Baseline, $\mathrm{g} \cdot \mathrm{cm}^{-2}($ mean $[S D])$ & $0.94(0.15)$ & $0.98(0.16)$ & $0.93(0.14)$ \\
\hline \multicolumn{4}{|c|}{ Change from baseline at Week 52, \% } \\
\hline LSM & -0.87 & -1.12 & -0.32 \\
\hline $95 \% \mathrm{Cl}$ & $(-1.39,-0.34)$ & $(-1.90,-0.33)$ & $(-0.86,0.23)$ \\
\hline \multicolumn{4}{|c|}{ LSM \% difference between treatments" } \\
\hline \multicolumn{4}{|l|}{ Versus GFF MDI 18/9.6 $\mu \mathrm{g}$} \\
\hline LSM & -0.55 & -0.81 & NA \\
\hline $95 \% \mathrm{Cl}$ & $(-1.30,0.21)$ & $(-1.79,0.16)$ & \\
\hline \multicolumn{4}{|l|}{ Versus BFF MDI 320/9.6 $\mu \mathrm{g}$} \\
\hline LSM & 0.25 & NA & Shown above \\
\hline $95 \% \mathrm{Cl}$ & $(-0.70,1.21)$ & & \\
\hline
\end{tabular}

BMD Bone mineral density, BFF Budesonide/formoterol fumarate, BGF Budesonide/glycopyrrolate/formoterol fumarate, CI Confidence interval, GFF Glycopyrrolate/ formoterol fumarate, $L$ Lumbar spine segment, $L S M$ Least squares mean, MDI Metered dose inhaler, NA Not applicable, SD Standard deviation, ${ }^{\#}$ non-inferiority was declared if the lower confidence bound for the percentage treatment difference was $>-2 \%$, not a pre-specified non-inferiority comparison

eyes) in LogMAR visual acuity or horizontal cup-to-disc ratio across the treatment groups at Week 52 (not shown).

Both ICS-containing treatment groups (BGF MDI and BFF MDI) had numerically greater proportions of patients relative to GFF MDI with Class $1(\geq 0.5)$ increases in $P$ score, and Class $2(\geq 1.0)$ increases in $C$ score (Table 5). BGF MDI also had numerically greater proportions of patients relative to BFF MDI and GFF MDI with Class 2 increases in NO score and $\mathrm{P}$ score. A numerically greater proportion of patients receiving GFF MDI had Class 1 and 2 increases in NC scores relative to BGF MDI and BFF MDI. Importantly, few patients $(\leq 8.2 \%)$ across treatment groups had a grade increase of $\geq 1.5$ (Class 3 ) in LOCS III scores of any scale at Week 52 (Table 5). Finally, few patients had an IOP $\geq 22 \mathrm{mmHg}$ or an increase from baseline $\geq 7 \mathrm{mmHg}$ in either eye, with similar proportions across the treatment groups (Table 5).

\section{Adverse events}

Overall, the majority of patients experienced at least one treatment-emergent $\mathrm{AE}$ (TEAE); most were mild or moderate in intensity and the majority were considered not treatment-related by the investigator (Table 6). The most commonly reported TEAEs overall were upper respiratory tract infection, bronchitis, COPD (recorded as a TEAE only if meeting the criteria for a serious TEAE), and urinary tract infection (Table 6). In general, the incidence and types of TEAEs were generally similar across treatment groups. COPD $(n=22 ; 4.8 \%)$ and pneumonia $(n=6 ; 1.3 \%)$ were the most frequently reported serious TEAEs; all others occurred in $\leq 2$ patients $(0.4 \%)$ overall. 
Table 3 Shifts in BMD T-scores" (baseline to Week 52; BMD population)

\begin{tabular}{|c|c|c|c|c|}
\hline & \multirow[t]{3}{*}{ Baseline T-score } & \multicolumn{3}{|c|}{ Post-baseline T-score } \\
\hline & & $\leq-2.5$ & $>-2.5$ and $\leq-1$ & $>-1$ \\
\hline & & Osteoporotic & Osteopenic & Normal \\
\hline \multicolumn{5}{|c|}{ Shifts in T-scores for lumbar spine BMD (L2-L4) } \\
\hline \multirow[t]{3}{*}{ BGF MDI 320/18/9.6 $\mu \mathrm{g}(N=128)^{\prime}$} & $\leq-2.5(n=0)$ & 0 & 0 & 0 \\
\hline & $>-2.5$ and $\leq-1 \quad(n=37)$ & 0 & $35(94.6)$ & $2(5.4)$ \\
\hline & $>-1(n=91)$ & 0 & $4(4.4)$ & $87(95.6)$ \\
\hline \multirow[t]{3}{*}{ BFF MDI 320/9.6 $\mu \mathrm{g}(N=57)^{\prime}$} & $\leq-2.5(n=0)$ & 0 & 0 & 0 \\
\hline & $>-2.5$ and $\leq-1 \quad(n=16)$ & 0 & $14(87.5)$ & $2(12.5)$ \\
\hline & $>-1(n=41)$ & 0 & $3(7.3)$ & $38(92.7)$ \\
\hline \multirow[t]{3}{*}{ GFF MDI 18/9.6 $\mu \mathrm{g}(N=123)^{n}$} & $\leq-2.5(n=0)$ & 0 & 0 & 0 \\
\hline & $>-2.5$ and $\leq-1 \quad(n=35)$ & 0 & $26(74.3)$ & $9(25.7)$ \\
\hline & $>-1(n=88)$ & 0 & $4(4.5)$ & $84(95.5)$ \\
\hline \multicolumn{5}{|l|}{ Shifts in T-scores for total hip BMD } \\
\hline \multirow[t]{3}{*}{ BGF MDI 320/18/9.6 $\mu \mathrm{g}(N=128)^{\prime}$} & $\leq-2.5(n=0)$ & 0 & 0 & 0 \\
\hline & $>-2.5$ and $\leq-1 \quad(n=52)$ & $3(5.8)$ & $45(86.5)$ & $4(7.7)$ \\
\hline & $>-1(n=76)$ & 0 & $5(6.6)$ & $71(93.4)$ \\
\hline \multirow[t]{3}{*}{ BFF MDI 320/9.6 $\mu \mathrm{g}(N=57)^{9}$} & $\leq-2.5(n=0)$ & 0 & 0 & 0 \\
\hline & $>-2.5$ and $\leq-1 \quad(n=18)$ & $1(5.6)$ & $16(88.9)$ & $1(5.6)$ \\
\hline & $>-1(n=39)$ & 0 & $5(12.8)$ & $34(87.2)$ \\
\hline \multirow[t]{3}{*}{ GFF MDI 18/9.6 $\mu \mathrm{g}(N=119)^{q}$} & $\leq-2.5(n=0)$ & 0 & 0 & 0 \\
\hline & $>-2.5$ and $\leq-1 \quad(n=52)$ & $1(1.9)$ & $50(96.2)$ & $1(1.9)$ \\
\hline & $>-1(n=67)$ & 0 & $4(6.0)$ & $63(94.0)$ \\
\hline
\end{tabular}

Data are $\mathrm{n}$ (\%). BMD Bone mineral density, BFF Budesonide/formoterol fumarate, BGF Budesonide/glycopyrrolate/formoterol fumarate, GFF Glycopyrrolate/ formoterol fumarate, $L$ Lumbar spine segment, $M D I$ Metered dose inhaler. ": shifts are from baseline to the worst post-baseline value, " $: N=$ number of patients with available data for both baseline and post-baseline T-scores (non-missing)

The incidence of AEs of special interest, including boneand ocular-related AEs, was low and similar across treatment groups. Overall, $61.1 \%(57.1-68.8 \%$ across treatment groups) and $55.6 \%(51.4-58.1 \%$ across treatment groups) of patients experienced TEAEs in the first 24 weeks and after Week 24 of the study, respectively (Additional file 1: Table S2). The pattern and frequency of individual TEAEs were generally similar across treatment groups during the first 24 weeks and after Week 24. During the first 24 weeks of the study, the most commonly reported TEAEs overall were upper respiratory tract infection, nasopharyngitis, and muscle spasms; after Week 24, the most commonly reported were upper respiratory tract infection, viral upper respiratory tract infection, and COPD (Additional file 1: Table S2).

None of the four deaths reported during the study were considered treatment-related by the investigator (three in the BGF MDI group resulted from cerebral infarction, respiratory fume inhalation disorder, and sepsis; one in the GFF MDI group resulted from myocardial ischemia). Six patients ( $n=3$, BGF MDI; $n=3$, GFF MDI) had events confirmed as MACE by the Clinical Endpoint Committee (four non-fatal myocardial infarctions and two cardiovascular deaths). The incidence of confirmed pneumonia was low overall $(n=11 ; 2.4 \%)$ and was highest in the GFF MDI group (3.4\%), followed by BGF MDI (2.1\%), and BFF MDI (1.1\%; Table 6).

\section{Efficacy endpoints}

BGF MDI increased time to first moderate/severe COPD exacerbation over the entire treatment period relative to GFF MDI and throughout the majority of the treatment period relative to BFF MDI (Fig. 2). The rate of moderate/severe exacerbations over 52 weeks was lowest in the BGF MDI group (0.59), followed by BFF MDI (0.72), and GFF MDI (0.81; Additional file 1: Table S3). Reductions from baseline in daily rescue salbutamol use, percentage of rescue-free days and changes from baseline in EXACT scores were comparable across treatments (Additional file 1: Table S3).

\section{Discussion}

This study evaluated the long-term safety and tolerability of BGF MDI, BFF MDI, and GFF MDI in patients with moderate-to-very severe COPD over 52 weeks (i.e., over the 24- 
Table 4 Primary and other ophthalmological endpoints" (ophthalmological population)

\begin{tabular}{|c|c|c|c|}
\hline & $\begin{array}{l}\text { BGF MDI } \\
320 / 18 / 9.6 \mu \mathrm{g} \\
(N=132)\end{array}$ & $\begin{array}{l}\text { BFF MDI } \\
320 / 9.6 \mu \mathrm{g} \\
(N=54)\end{array}$ & $\begin{array}{l}\text { GFF MDI } \\
18 / 9.6 \mu \mathrm{g} \\
(N=125)\end{array}$ \\
\hline \multicolumn{4}{|l|}{ LOCS III P score } \\
\hline$n$ & 218 & 98 & 184 \\
\hline Baseline (mean [SD]) & $0.381(0.880)$ & $0.397(0.650)$ & $0.308(0.567)$ \\
\hline \multicolumn{4}{|c|}{$\begin{array}{l}\text { Change from baseline to Week } 52 \\
\text { (primary ophthalmological endpoint) }\end{array}$} \\
\hline LSM & 0.153 & 0.022 & 0.026 \\
\hline $95 \% \mathrm{Cl}$ & $(0.079,0.227)$ & $(-0.090,0.135)$ & $(-0.055,0.106)$ \\
\hline \multicolumn{4}{|c|}{ LSM difference between treatments } \\
\hline \multicolumn{4}{|c|}{ Versus GFF MDI 18/9.6 $\mu g^{q}$} \\
\hline LSM & 0.127 & -0.003 & \multirow[t]{2}{*}{ NA } \\
\hline $95 \% \mathrm{Cl}$ & $(0.017,0.237)$ & $(-0.142,0.135)$ & \\
\hline \multicolumn{4}{|c|}{ Versus BFF MDI 320/9.6 $\mu \mathrm{g}^{\S}$} \\
\hline LSM & 0.130 & \multirow[t]{2}{*}{ NA } & \multirow[t]{2}{*}{ Shown above } \\
\hline $95 \% \mathrm{Cl}$ & $(-0.004,0.265)$ & & \\
\hline \multicolumn{4}{|l|}{ LOCS III NO score } \\
\hline$n$ & 220 & 98 & 187 \\
\hline Baseline (mean [SD]) & $2.447(1.082)$ & $2.336(0.886)$ & $2.309(1.060)$ \\
\hline \multicolumn{4}{|c|}{ Change from baseline to Week 52} \\
\hline LSM & \multirow{2}{*}{$\begin{array}{l}0.255 \\
(0.170,0.340)\end{array}$} & \multirow{2}{*}{$\begin{array}{l}0.186 \\
(0.059,0.314)\end{array}$} & \multirow{2}{*}{$\begin{array}{l}0.047 \\
(-0.045,0.138)\end{array}$} \\
\hline $95 \% \mathrm{Cl}$ & & & \\
\hline \multicolumn{4}{|c|}{ LSM difference between treatments ${ }^{\S}$} \\
\hline \multicolumn{4}{|c|}{ Versus GFF MDI 18/9.6 $\mu \mathrm{g}$} \\
\hline LSM & 0.208 & 0.140 & \multirow[t]{2}{*}{ NA } \\
\hline $95 \% \mathrm{Cl}$ & $(0.084,0.333)$ & $(-0.017,0.297)$ & \\
\hline \multicolumn{4}{|c|}{ Versus BFF MDI 320/9.6 $\mu \mathrm{g}$} \\
\hline LSM & 0.069 & \multirow[t]{2}{*}{ NA } & \multirow[t]{2}{*}{ Shown above } \\
\hline $95 \% \mathrm{Cl}$ & $(-0.084,0.222)$ & & \\
\hline \multicolumn{4}{|l|}{ LOCS III NC score } \\
\hline$n$ & 219 & 98 & 187 \\
\hline Baseline (mean [SD]) & $2.290(1.137)$ & $2.287(0.951)$ & $2.178(0.858)$ \\
\hline \multicolumn{4}{|c|}{ Change from baseline to Week 52} \\
\hline LSM & 0.130 & 0.142 & 0.163 \\
\hline $95 \% \mathrm{Cl}$ & $(0.050,0.209)$ & $(0.022,0.263)$ & $(0.077,0.248)$ \\
\hline \multicolumn{4}{|c|}{ LSM difference between treatments ${ }^{\S}$} \\
\hline \multicolumn{4}{|c|}{ Versus GFF MDI 18/9.6 $\mu \mathrm{g}$} \\
\hline LSM & -0.033 & -0.021 & \multirow[t]{2}{*}{ NA } \\
\hline $95 \% \mathrm{Cl}$ & $(-0.150,0.084)$ & $(-0.169,0.128)$ & \\
\hline \multicolumn{4}{|c|}{ Versus BFF MDI 320/9.6 $\mu \mathrm{g}$} \\
\hline LSM & -0.013 & \multirow[t]{2}{*}{ NA } & Shown above \\
\hline $95 \% \mathrm{Cl}$ & $(-0.157,0.132)$ & & \\
\hline LOCS III C score & & & \\
\hline$n$ & 218 & 98 & 187 \\
\hline Baseline (mean [SD]) & $0.832(1.189)$ & $0.801(0.976)$ & $0.727(1.060)$ \\
\hline
\end{tabular}


Table 4 Primary and other ophthalmological endpoints ${ }^{\#}$ (ophthalmological population) (Continued)

\begin{tabular}{|c|c|c|c|}
\hline & $\begin{array}{l}\text { BGF MDI } \\
320 / 18 / 9.6 \mu \mathrm{g} \\
(N=132)\end{array}$ & $\begin{array}{l}\text { BFF MDI } \\
320 / 9.6 \mu \mathrm{g} \\
(N=54)\end{array}$ & $\begin{array}{l}\text { GFF MDI } \\
18 / 9.6 \mu \mathrm{g} \\
(N=125)\end{array}$ \\
\hline \multicolumn{4}{|c|}{ Change from baseline to Week 52} \\
\hline LSM & \multirow{3}{*}{$\begin{array}{l}0.105 \\
(0.022,0.187)\end{array}$} & \multirow{3}{*}{$\begin{array}{l}0.170 \\
(0.047,0.294)\end{array}$} & \multirow{3}{*}{$\begin{array}{l}0.067 \\
(-0.022,0.155)\end{array}$} \\
\hline $95 \% \mathrm{Cl}$ & & & \\
\hline LSM difference between treatments $^{\S}$ & & & \\
\hline \multicolumn{4}{|c|}{ Versus GFF MDI 18/9.6 $\mu \mathrm{g}$} \\
\hline LSM & 0.038 & 0.103 & \multirow[t]{2}{*}{ NA } \\
\hline $95 \% \mathrm{Cl}$ & $(-0.083,0.159)$ & $(-0.049,0.256)$ & \\
\hline \multicolumn{4}{|c|}{ Versus BFF MDI 320/9.6 $\mu \mathrm{g}$} \\
\hline LSM & -0.065 & \multirow[t]{2}{*}{ NA } & \multirow[t]{2}{*}{ Shown above } \\
\hline $95 \% \mathrm{Cl}$ & $(-0.214,0.083)$ & & \\
\hline \multicolumn{4}{|l|}{$\mathrm{IOP}, \mathrm{mmHg}$} \\
\hline n & 228 & 100 & 203 \\
\hline Baseline (mean [SD]) & $14.482(2.908)$ & $15.490(2.740)$ & $14.978(2.971)$ \\
\hline \multicolumn{4}{|c|}{ Change from baseline to Week 52} \\
\hline LSM & 0.670 & 0.178 & 0.680 \\
\hline $95 \% \mathrm{Cl}$ & $(0.330,1.010)$ & $(-0.339,0.696)$ & $(0.321,1.039)$ \\
\hline \multicolumn{4}{|c|}{ LSM difference between treatments ${ }^{\S}$} \\
\hline \multicolumn{4}{|c|}{ Versus GFF MDI 18/9.6 $\mu \mathrm{g}$} \\
\hline LSM & -0.010 & -0.502 & \multirow[t]{2}{*}{ NA } \\
\hline $95 \% \mathrm{Cl}$ & $(-0.505,0.486)$ & $(-1.131,0.128)$ & \\
\hline \multicolumn{4}{|c|}{ Versus BFF MDI 320/9.6 $\mu \mathrm{g}$} \\
\hline LSM & 0.492 & \multirow[t]{2}{*}{ NA } & \multirow[t]{2}{*}{ Shown above } \\
\hline $95 \% \mathrm{Cl}$ & $(-0.129,1.113)$ & & \\
\hline
\end{tabular}

BFF Budesonide/formoterol fumarate, BGF Budesonide/glycopyrrolate/formoterol fumarate, C Cortical cataract, CI Confidence interval, GFF Glycopyrrolate/ formoterol fumarate, IOP Intraocular pressure, LOCS III Lens opacities classification system III, LSM Least squares mean, MDI Metered dose inhaler, NA Not applicable, NC Nuclear color, NO Nuclear opalescence, $P$ Posterior subcapsular cataract, SD Standard deviation. ": data presented are across eyes (irrespective of person) such that $n=$ total number of eyes assessed, " : non-inferiority was declared if the upper confidence bound for the treatment difference was $<0.5$, ${ }^{\S}$ : not a pre-specified non-inferiority comparison

week KRONOS study [2] and a 28-week extension). Because long-term use of ICS can be associated with increased risk of fractures and cataracts [16-19], BMD and ophthalmological assessments were selected as primary safety endpoints. To the best of our knowledge, this is the first study to specifically assess bone and ocular safety outcomes in patients with COPD following treatment with an ICS/LAMA/LABA triple FDC therapy.

Overall, changes in BMD and ophthalmological assessments were small in this study, and BGF MDI was non-inferior to the non-ICS-containing treatment (GFF MDI) for both primary endpoints. The effects of BGF MDI on BMD and ophthalmological assessments were similar in magnitude to those previously reported for budesonide/formoterol MDI in patients with moderate-to-very severe COPD [11]. Our study did not show evidence of any clinically meaningful ICS-induced changes in BMD or LOCS III scores. For instance, shifts in BMD T-scores from normal to osteopenic levels $(>-2.5$ and $\leq-1)$ were similar in the BGF MDI and
GFF MDI groups. Few patients across treatment groups experienced a worsening $\mathrm{T}$-score for lumbar spine $\mathrm{BMD}$ relative to baseline, and no patients shifted to a lumbar spine Tscore $\leq-2.5$ (indicative of osteoporosis) after 52 weeks of treatment. Although five patients (three receiving BGF MDI and one each in the BFF MDI and GFF MDI groups) had a total hip T-score $\leq-2.5$ at Week 52 , each of these patients had a baseline T-score $<-2$, suggesting that this did not represent a major decline in BMD over the study period.

For LOCS III scores, no consistent changes attributable to ICS were seen in this study, and the incidence of Class 3 shifts was low $(\leq 8.2 \%)$. While Class 1 and 2 increases were more frequent overall, Class 3 shifts are the most likely to represent a clinically meaningful treatment effect, rather than a result of aging or inter-assessment variability [21]. LOCS III shifts have been observed in patients with COPD receiving no active treatments, with a previous study finding that $18.6,6.0$, and $2.2 \%$ of patients 
Table 5 Shifts in LOCS III scores and IOP (Week 52; ophthalmological population)

\begin{tabular}{|c|c|c|c|}
\hline & BGF MDI 320/18/9.6 $\mu \mathrm{g}(N=132)$ & BFF MDI 320/9.6 $\mu \mathrm{g}(N=54)$ & GFF MDI 18/9.6 $\mu \mathrm{g}(N=125)$ \\
\hline \multicolumn{4}{|c|}{ Proportion of patients with increases in LOCS III P score, $n(\%)$} \\
\hline$n$ & 111 & 49 & 94 \\
\hline Class 1 ( $\geq 0.5$ unit) & $16(14.4)$ & $6(12.2)$ & $7(7.4)$ \\
\hline Class 2 ( $\geq 1.0$ unit) & $12(10.8)$ & $3(6.1)$ & $5(5.3)$ \\
\hline Class 3 ( $\geq 1.5$ units) & $8(7.2)$ & 0 & $4(4.3)$ \\
\hline \multicolumn{4}{|c|}{ Proportion of patients with increases in LOCS III NO score, $n(\%)$} \\
\hline$n$ & 111 & 49 & 95 \\
\hline Class 1 ( $\geq 0.5$ unit) & $40(36.0)$ & $16(32.7)$ & $33(34.7)$ \\
\hline Class 2 ( $\geq 1.0$ unit) & $19(17.1)$ & $5(10.2)$ & $11(11.6)$ \\
\hline Class 3 ( $\geq 1.5$ units) & $6(5.4)$ & $2(4.1)$ & $3(3.2)$ \\
\hline \multicolumn{4}{|c|}{ Proportion of patients with increases in LOCS III NC score, $n$ (\%) } \\
\hline$n$ & 111 & 49 & 95 \\
\hline Class 1 ( $\geq 0.5$ unit) & $30(27.0)$ & $12(24.5)$ & $32(33.7)$ \\
\hline Class 2 ( $\geq 1.0$ unit) & $11(9.9)$ & $4(8.2)$ & $14(14.7)$ \\
\hline Class 3 ( $\geq 1.5$ units) & $5(4.5)$ & $1(2.0)$ & $3(3.2)$ \\
\hline \multicolumn{4}{|c|}{ Proportion of patients with increases in LOCS III C score, $n(\%)$} \\
\hline$n$ & 110 & 49 & 95 \\
\hline Class 1 ( $\geq 0.5$ unit) & $25(22.7)$ & $10(20.4)$ & $17(17.9)$ \\
\hline Class 2 ( $\geq 1.0$ unit) & $15(13.6)$ & $6(12.2)$ & $5(5.3)$ \\
\hline Class 3 ( $\geq 1.5$ units) & $8(7.3)$ & $4(8.2)$ & $4(4.2)$ \\
\hline \multicolumn{4}{|c|}{ Proportion of patients with $\mathrm{IOP} \geq 22 \mathrm{mmHg}$ or increase in IOP of $\geq 7 \mathrm{mmHg}, n(\%)^{\#}$} \\
\hline$n$ & 114 & 50 & 103 \\
\hline$\geq 22 \mathrm{mmHg}$ & $2(1.8)$ & $3(6.0)$ & $4(3.9)$ \\
\hline$\geq 7 \mathrm{mmHg}$ increase & $5(4.4)$ & $2(4.0)$ & $3(2.9)$ \\
\hline
\end{tabular}

BFF Budesonide/formoterol fumarate, BGF Budesonide/glycopyrrolate/formoterol fumarate, C Cortical cataract, GFF Glycopyrrolate/formoterol fumarate, IOP Intraocular pressure, LOCS III Lens opacities classification system III, MDI Metered dose inhaler, NC Nuclear color, NO Nuclear opalescence, $P$ Posterior subcapsular cataract. ": in either eye

receiving a placebo had Class 1,2 , and 3 increases, respectively, in $\mathrm{P}, \mathrm{NO}$, and/or $\mathrm{C}$ scores after 26 weeks [21]. Since our study did not have a placebo arm, we are unable to assess age-related deterioration in BMD and LOCS III scores, and many of the changes seen in BMD and ocular endpoints may be attributable to normal aging and COPD-related changes, rather than to any treatment component of BGF MDI.

The overall incidence of bone-related and ocular AEs was low and similar across treatment groups, consistent with other large randomized controlled trials in COPD that found no increase in rates of bone-related or ocular AEs in ICS treatment groups [14, 22].

Notably, after 52 weeks of treatment, pneumonia incidence was similar across groups, and slightly lower in the BGF MDI group relative to GFF MDI, confirming the findings of KRONOS [2] that the addition of budesonide to GFF MDI did not result in an apparent increased risk of pneumonia. In addition, there were no cumulative adverse effects of treatment over time as the incidence and types of TEAEs, including pneumonia, were generally similar in the first 24 weeks of the study and after Week 24. Overall, all treatments were well tolerated with no new or unexpected safety findings, and the safety profile of BGF MDI was similar to the well-characterized safety profiles of GFF MDI [23-25] and approved budesonide/formoterol combinations $[11,26]$.

In addition to the primary and secondary safety objectives of the study, several efficacy endpoints were also assessed. Although no inferential analyses were planned or performed, the finding that BGF MDI reduced the rate of moderate/severe exacerbations compared to GFF MDI and BFF MDI is in alignment with results from the 24-week KRONOS study [2], as well as previous studies comparing triple and dual FDC therapies over 52 weeks [3, 27, 28]. While all three treatments reduced rescue medication use and EXACT scores over 52 weeks, these 
Table 6 Summary of TEAEs (safety population)

\begin{tabular}{|c|c|c|c|c|}
\hline TEAEs, $n(\%)$ & $\begin{array}{l}\text { BGF MDI } \\
320 / 18 / 9.6 \mu \mathrm{g} \\
(N=194)\end{array}$ & $\begin{array}{l}\text { BFF MDI } \\
320 / 9.6 \mu \mathrm{g} \\
(N=88)\end{array}$ & $\begin{array}{l}\text { GFF MDI } \\
18 / 9.6 \mu \mathrm{g} \\
(N=174)\end{array}$ & $\begin{array}{l}\text { All } \\
\text { patients } \\
(N=456)\end{array}$ \\
\hline Patients with $\geq 1$ TEAE & $144(74.2)$ & $64(72.7)$ & $133(76.4)$ & $341(74.8)$ \\
\hline Mild & $50(25.8)$ & $27(30.7)$ & $46(26.4)$ & $123(27.0)$ \\
\hline Moderate & $63(32.5)$ & $29(33.0)$ & $65(37.4)$ & $157(34.4)$ \\
\hline Severe & $31(16.0)$ & $8(9.1)$ & $22(12.6)$ & $61(13.4)$ \\
\hline $\begin{array}{l}\text { Patients with treatment-related } \\
\text { TEAEs }\end{array}$ & $35(18.0)$ & $17(19.3)$ & $29(16.7)$ & $81(17.8)$ \\
\hline Patients with serious TEAEs ${ }^{\S}$ & $33(17.0)$ & $7(8.0)$ & $22(12.6)$ & $62(13.6)$ \\
\hline $\mathrm{COPD}^{+}$ & $12(6.2)$ & $1(1.1)$ & $9(5.2)$ & $22(4.8)$ \\
\hline Pneumonia & $2(1.0)$ & 0 & $4(2.3)$ & $6(1.3)$ \\
\hline $\begin{array}{l}\text { Patients with treatment-related serious } \\
\text { TEAEN\$\$\$ }\end{array}$ & $2(1.0)$ & 0 & $2(1.1)$ & $4(0.9)$ \\
\hline $\begin{array}{l}\text { Patients with TEAEs leading to early } \\
\text { discontinuation }\end{array}$ & $16(8.2)$ & $6(6.8)$ & $12(6.9)$ & $34(7.5)$ \\
\hline Patients with confirmed MACE" & $3(1.5)$ & 0 & $3(1.7)$ & $6(1.3)$ \\
\hline Patients with confirmed pneumonia" & $4(2.1)$ & $1(1.1)$ & $6(3.4)$ & $11(2.4)$ \\
\hline Deaths (all causes) & $3(1.5)$ & 0 & $1(0.6)$ & $4(0.9)$ \\
\hline \multicolumn{5}{|l|}{$\begin{array}{l}\text { TEAEs occurring in } \geq 4 \% \text { of patients in } \\
\text { any treatment arm }\end{array}$} \\
\hline Upper respiratory tract infection & $18(9.3)$ & $6(6.8)$ & $18(10.3)$ & $42(9.2)$ \\
\hline Bronchitis & $12(6.2)$ & $2(2.3)$ & $8(4.6)$ & $22(4.8)$ \\
\hline $\mathrm{COPD}^{+}$ & $12(6.2)$ & $1(1.1)$ & $9(5.2)$ & $22(4.8)$ \\
\hline Urinary tract infection & $10(5.2)$ & $5(5.7)$ & $6(3.4)$ & $21(4.6)$ \\
\hline Muscle spasms & $6(3.1)$ & $9(10.2)$ & $5(2.9)$ & $20(4.4)$ \\
\hline Viral upper respiratory tract infection & $9(4.6)$ & $6(6.8)$ & $5(2.9)$ & $20(4.4)$ \\
\hline Sinusitis & $11(5.7)$ & $2(2.3)$ & $6(3.4)$ & $19(4.2)$ \\
\hline Hypertension & $8(4.1)$ & $4(4.5)$ & $6(3.4)$ & $18(3.9)$ \\
\hline Back pain & $9(4.6)$ & $1(1.1)$ & $7(4.0)$ & $17(3.7)$ \\
\hline Nasopharyngitis & $7(3.6)$ & $4(4.5)$ & $6(3.4)$ & $17(3.7)$ \\
\hline Diarrhea & $5(2.6)$ & $2(2.3)$ & $9(5.2)$ & $16(3.5)$ \\
\hline Dyspnea & $4(2.1)$ & $5(5.7)$ & $5(2.9)$ & $14(3.1)$ \\
\hline Pneumonia & $5(2.6)$ & $1(1.1)$ & $8(4.6)$ & $14(3.1)$ \\
\hline Dysphonia & $6(3.1)$ & $5(5.7)$ & $2(1.1)$ & $13(2.9)$ \\
\hline \multicolumn{5}{|l|}{$\begin{array}{l}\text { Bone- and ocular-related TEAEs } \\
\text { occurring in } \geq 1 \% \text { of patients }\end{array}$} \\
\hline Cataract & $6(3.1)$ & $2(2.3)$ & 0 & $8(1.8)$ \\
\hline IOP increased & $2(1.0)$ & $1(1.1)$ & $4(2.3)$ & $7(1.5)$ \\
\hline Osteoarthritis & $2(1.0)$ & 0 & $4(2.3)$ & $6(1.3)$ \\
\hline Osteoporosis & $2(1.0)$ & $1(1.1)$ & $2(1.1)$ & $5(1.1)$ \\
\hline
\end{tabular}

BFF Budesonide/formoterol fumarate, BGF Budesonide/glycopyrrolate/formoterol fumarate, COPD Chronic obstructive pulmonary disease, GFF Glycopyrrolate/ formoterol fumarate, IOP Intraocular pressure, MACE Major adverse cardiovascular event, MDI Metered dose inhaler, TEAE Treatment-emergent adverse event. ": possibly, probably, or definitely related in the opinion of the investigator, " ${ }^{\natural}$ : confirmed by clinical endpoint committee, ${ }^{\varsigma}$ : TEAEs were classified as serious in the opinion of the investigator if they resulted in hospitalization or substantial disruption of the ability to conduct normal life functions, or were life-threatening/fatal), ${ }^{+}$: classified as a TEAE only if meeting criteria for a serious TEAE

findings should be interpreted in the context of the smaller sample size relative to the full KRONOS population.
One limitation of our study was that it excluded patients who had a baseline $\mathrm{T}$-score $<-2.5$, which may have led to the exclusion of patients with the greatest 


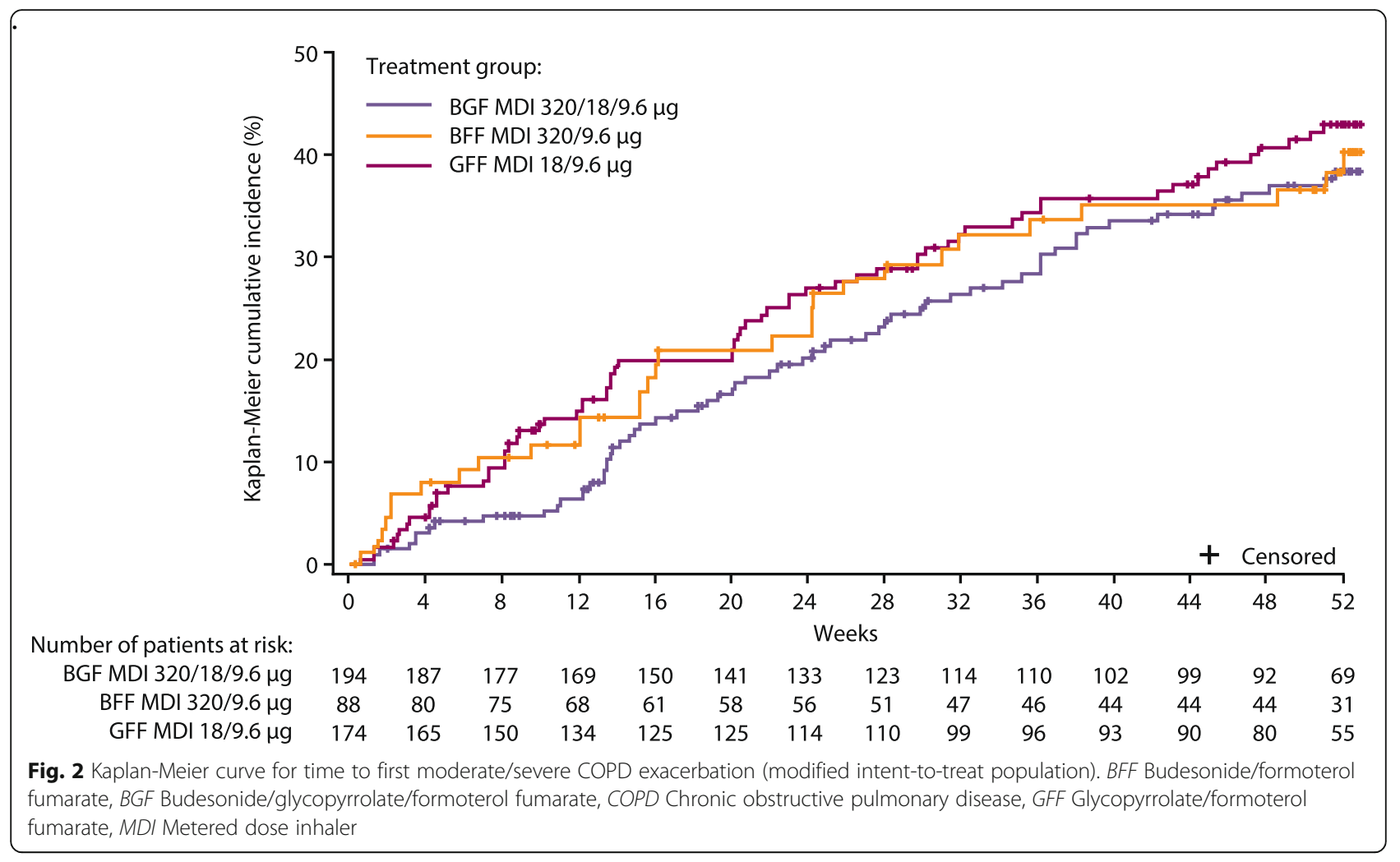

risk profile for developing adverse events related to osteoporosis. However, the probability of detecting a change or decline in BMD in such patients would likely be limited. Strengths included the one-year study duration, the use of corresponding dual and triple therapies, assessments by LOCS III-certified ocular clinicians, and an adjudication committee to assess cardiovascular MACE events and pneumonia AEs using standardized criteria.

\section{Conclusions}

Overall, this study found no clinically meaningful differences in BMD or ophthalmological safety assessments over 52 weeks after treatment with ICS-containing therapies BGF MDI and BFF MDI compared to the LAMA/LABA GFF MDI. All three treatments were well tolerated over 52 weeks with no new or unexpected safety findings, and the incidence of confirmed pneumonia was low and similar between BGF MDI and GFF MDI. In addition, there was no evidence of cumulative AEs, based on the incidence and types of AEs occurring in the first 24 weeks compared to after Week 24. The benefit of BGF MDI versus dual therapies on exacerbations was maintained when treatment was continued for up to 52 weeks. These findings provide additional evidence for the long-term tolerability of BGF MDI in patients with moderate-to-very severe COPD.

\section{Additional file}

Additional file 1: Supplementary methods. Table S1. Important changes to methods after trial commencement. Table S2. TEAEs by time period (extension study safety population). Table S3. Efficacy endpoints (over 52 weeks; modified intent-to-treat population). Additional file Figure S1. Study design. Additional file Figure S2. Percent change from baseline in BMD of the lumbar spine (L2-L4) and total hip at Week 52 (BMD population). Additional file Figure S3. Change from baseline in LOCS III scores at Week 52 (ophthalmological population). (DOCX $325 \mathrm{~kb}$ )

\section{Abbreviations}

AE: Adverse event; BFF: Budesonide/formoterol fumarate; BGF: Budesonide/ glycopyrrolate/formoterol fumarate; BMD: Bone mineral density; C: Cortical cataract; CAT: COPD Assessment Test; Cl: Confidence interval; COPD: Chronic obstructive pulmonary disease; DPI: Dry powder inhaler; EXACT: Exacerbations of Chronic Pulmonary Disease Tool; EXACT-RS: EXACT-Respiratory Symptom; FDC: Fixed-dose combination; $\mathrm{FEV}_{1}$ : Forced expiratory volume in $1 \mathrm{~s}$; GFF: Glycopyrrolate/formoterol fumarate; ICS: Inhaled corticosteroid; IOP: Intraocular pressure; LABA: Long-acting $\beta_{2}$-agonist; LAMA: Long-acting antimuscarinic antagonist; LOCS III: Lens opacities classification system III; LogMAR: Logarithm of the minimum angle of resolution; LSM: Least squares mean; MACE: Major adverse cardiovascular event; MDI: Metered dose inhaler; mITT: Modified intent-to-treat; NA: Not applicable; NC: Nuclear color; NO: Nuclear opalescence; P: Posterior subcapsular cataract; SD: Standard deviation

\section{Acknowledgments}

The authors would like to thank all of the volunteers, the team of investigators and the research and operations staff involved in this study. Medical writing support, under the direction of the authors, was provided by Julia King, PhD, of CMC Connect, a division of McCann Health Medical Communications Ltd., Glasgow, UK, which was funded by AstraZeneca, Gaithersburg, USA, in accordance with Good Publication Practice (GPP3) guidelines [29]. 


\section{Author contributions}

PD made substantial contributions to the conception and design of the work. EMK, GTF, MM, and KDeA made substantial contributions to the acquisition of data. MM, KDeA, and PD made substantial contributions to the analysis of data. EMK, GTF, MM, KDeA, and PD made substantial contributions to the interpretation of data. All authors were involved in the preparation and review of the manuscript, approved the final version to be submitted, and agree to be accountable for all aspects of the work, in ensuring that questions related to the accuracy or integrity of any part of the work are appropriately investigated and resolved.

\section{Funding}

This study was supported by Pearl - a member of the AstraZeneca Group. The funder of the study was involved in study design, data collection, data analysis, data interpretation, and writing of the report.

\section{Availability of data and materials}

The datasets used and analyzed during the current study are available on reasonable request in accordance with AstraZeneca's data sharing policy described at https://astrazenecagrouptrials.pharmacm.com/ST/ Submission/Disclosure

\section{Ethics approval and consent to participate}

The informed consent form and study protocol were approved by an institutional review board (Schulman Associates; approval number 201503097), and patients provided written informed consent for both KRONOS and the extension study prior to screening.

\section{Consent for publication}

Not applicable.

\section{Competing interests}

EMK has served on advisory boards, speaker panels or received travel reimbursement from Amphastar, AstraZeneca, Boehringer Ingelheim, Cipla, Forest, GlaxoSmithKline, Mylan, Novartis, Oriel, Pearl - a member of the AstraZeneca Group, Sunovion, Teva, and Theravance. He has conducted multicenter clinical trials for $\sim 40$ pharmaceutical companies. GTF reports grants, personal fees, and non-financial support from AstraZeneca during the conduct of the study; grants, personal fees, and non-financial support from AstraZeneca, Boehringer Ingelheim, Novartis, Pearl - a member of the AstraZeneca Group, and Sunovion; grants and personal fees from Theravance; and personal fees from Circassia, GlaxoSmithKline, Innoviva, Mylan, and Verona, outside the submitted work. MM, KDeA, and PD are employees of Pearl - a member of the AstraZeneca Group.

\section{Author details}

${ }^{1}$ Clinical Research Institute of Southern Oregon, 3860 Crater Lake Avenue, Medford, OR 97504, USA. ${ }^{2}$ Pulmonary Research Institute of Southeast Michigan, Farmington Hills, MI, USA. ${ }^{3}$ Formerly of AstraZeneca, Morristown, NJ, USA. ${ }^{4}$ AstraZeneca, Durham, NC, USA.

\section{Received: 23 May 2019 Accepted: 4 July 2019}

\section{Published online: 29 July 2019}

\section{References}

1. Global Initiative for Chronic Obstructive Lung Disease. 2019 Report: Global Strategy for Prevention, Diagnosis and Management of COPD 2019. https:// goldcopd.org/. Accessed 11 Apr 2019.

2. Ferguson GT, Rabe KF, Martinez FJ, Fabbri LM, Wang C, Ichinose M, et al. Triple therapy with budesonide/glycopyrrolate/formoterol fumarate with co-suspension delivery technology versus dual therapies in chronic obstructive pulmonary disease (KRONOS): a double-blind, parallel-group, multicentre, phase 3 randomised controlled trial. Lancet Respir Med. 2018;6:747-58.

3. Lipson DA, Barnhart F, Brealey N, Brooks J, Criner GJ, Day NC, et al. Once-daily single-inhaler triple versus dual therapy in patients with COPD. N Engl J Med. 2018;378:1671-80.

4. Mannino DM, Watt G, Hole D, Gillis C, Hart C, McConnachie A, et al. The natural history of chronic obstructive pulmonary disease. Eur Respir J. 2006; 27:627-43.
5. Klein R, Klein BEK. The prevalence of age-related eye diseases and visual impairment in aging: current estimates. Invest Ophthalmol Vis Sci. 2013;54: ORSF5-ORSF13.

6. Warming L, Hassager C, Christiansen C. Changes in bone mineral density with age in men and women: a longitudinal study. Osteoporos Int. 2002;13: 105-12.

7. Soriano JB, Visick GT, Muellerova H, Payvandi N, Hansell AL. Patterns of comorbidities in newly diagnosed COPD and asthma in primary care. Chest. 2005;128:2099-107.

8. Chatila WM, Thomashow BM, Minai OA, Criner GJ, Make BJ. Comorbidities in chronic obstructive pulmonary disease. Proc Am Thorac Soc. 2008;5:549-55.

9. Milkowska-Dymanowska J, Bialas AJ, Zalewska-Janowska A, Górski P, Piotrowski WJ. Underrecognized comorbidities of chronic obstructive pulmonary disease. Int J Chron Obstruct Pulmon Dis. 2015;10:1331-41.

10. Okazaki R, Watanabe R, Inoue D. Osteoporosis associated with chronic obstructive pulmonary disease. J Bone Metab. 2016;23:111-20.

11. Rennard SI, Tashkin DP, McElhattan J, Goldman M, Ramachandran S, Martin UJ, et al. Efficacy and tolerability of budesonide/formoterol in one hydrofluoroalkane pressurized metered-dose inhaler in patients with chronic obstructive pulmonary disease: results from a 1-year randomized controlled clinical trial. Drugs. 2009;69:549-65.

12. Ferguson GT, Calverley PMA, Anderson JA, Jenkins CR, Jones PW, Willits LR, et al. Prevalence and progression of osteoporosis in patients with COPD: results from the TOwards a revolution in COPD health study. Chest. 2009; 136:1456-65.

13. Johnell O, Pauwels R, Löfdahl C-G, Laitinen LA, Postma DS, Pride NB, et al. Bone mineral density in patients with chronic obstructive pulmonary disease treated with budesonide Turbuhaler ${ }^{\oplus}$. Eur Respir J. 2002;19:1058-63.

14. Pauwels RA, Löfdahl C-G, Laitinen LA, Schouten JP, Postma DS, Pride NB, et al. Long-term treatment with inhaled budesonide in persons with mild chronic obstructive pulmonary disease who continue smoking. For the European Respiratory Society study on chronic obstructive pulmonary disease. N Engl J Med. 1999;340:1948-53.

15. Wang JJ, Rochtchina E, Tan AG, Cumming RG, Leeder SR, Mitchell P. Use of inhaled and oral corticosteroids and the long-term risk of cataract. Ophthalmology. 2009;116:652-7.

16. Ernst $P$, Baltzan M, Deschênes J, Suissa S. Low-dose inhaled and nasal corticosteroid use and the risk of cataracts. Eur Respir J. 2006;27:1168-74.

17. Cumming RG, Mitchell $P$, Leeder SR. Use of inhaled corticosteroids and the risk of cataracts. N Engl J Med. 1997;337:8-14.

18. Loke YK, Cavallazzi R, Singh S. Risk of fractures with inhaled corticosteroids in COPD: systematic review and meta-analysis of randomised controlled trials and observational studies. Thorax. 2011;66:699-708.

19. Gonzalez AV, Coulombe J, Ernst P, Suissa S. Long-term use of inhaled corticosteroids in COPD and the risk of fracture. Chest. 2018;153:321-8.

20. Leidy NK, Murray LT. Patient-reported outcome (PRO) measures for clinical trials of COPD: the EXACT and E-RS. COPD. 2013;10:393-8.

21. Maspero J, Cherrez I, Doherty DE, Tashkin DP, Kuna P, Kuo W-L, et al. Appraisal of lens opacity with mometasone furoate/formoterol fumarate combination in patients with COPD or asthma. Respir Med. 2014;108:1355-62.

22. Calverley PMA, Anderson JA, Celli B, Ferguson GT, Jenkins C, Jones PW, et al. Salmeterol and fluticasone propionate and survival in chronic obstructive pulmonary disease. N Engl J Med. 2007;356:775-89.

23. Martinez FJ, Rabe KF, Ferguson GT, Fabbri LM, Rennard S, Feldman GJ, et al. Efficacy and safety of glycopyrrolate/formoterol metered dose inhaler formulated using co-suspension delivery technology in patients with COPD. Chest. 2017;151:340-57.

24. Hanania NA, Tashkin DP, Kerwin EM, Donohue JF, Denenberg M, O'Donnell $D E$, et al. Long-term safety and efficacy of glycopyrrolate/formoterol metered dose inhaler using novel Co-Suspension ${ }^{\text {TM }}$ Delivery Technology in patients with chronic obstructive pulmonary disease. Respir Med. 2017;126: 105-15.

25. Lipworth BJ, Collier DJ, Gon Y, Zhong N, Nishi K, Chen R, et al. Improved lung function and patient-reported outcomes with co-suspension delivery technology glycopyrrolate/formoterol fumarate metered dose inhaler in COPD: a randomized phase III study conducted in Asia, Europe, and the USA. Int J Chron Obstruct Pulmon Dis. 2018;13:2969-84.

26. Szafranski W, Cukier A, Ramirez A, Menga G, Sansores R, Nahabedian S, et al. Efficacy and safety of budesonide/formoterol in the management of chronic obstructive pulmonary disease. Eur Respir J. 2003;21:74-81. 
27. Papi A, Vestbo J, Fabbri L, Corradi M, Prunier H, Cohuet G, et al. Extrafine inhaled triple therapy versus dual bronchodilator therapy in chronic obstructive pulmonary disease (TRIBUTE): a double-blind, parallel group, randomised controlled trial. Lancet. 2018:391:1076-84.

28. Singh D, Papi A, Corradi M, Pavlišová I, Montagna I, Francisco C, et al. Single inhaler triple therapy versus inhaled corticosteroid plus long-acting $\beta_{2^{-}}$ agonist therapy for chronic obstructive pulmonary disease (TRILOGY): a double-blind, parallel group, randomised controlled trial. Lancet. 2016;388: 963-73.

29. Battisti WP, Wager E, Baltzer L, Bridges D, Cairns A, Carswell Cl, et al. Good publication practice for communicating company-sponsored medical research: GPP3. Ann Intern Med. 2015;163:461-4.

\section{Publisher's Note}

Springer Nature remains neutral with regard to jurisdictional claims in published maps and institutional affiliations.

Ready to submit your research? Choose BMC and benefit from:

- fast, convenient online submission

- thorough peer review by experienced researchers in your field

- rapid publication on acceptance

- support for research data, including large and complex data types

- gold Open Access which fosters wider collaboration and increased citations

- maximum visibility for your research: over $100 \mathrm{M}$ website views per year

At BMC, research is always in progress.

Learn more biomedcentral.com/submissions 